\title{
Design and Building of Liquid Electrostatic Experimental Equipment
}

\author{
Teng Xu, Hua Xie ${ }^{a}$, Guowei Zhao \\ School of Safety Engineering, Shenyang Aerospace University, Shenyang 110136, China \\ aemail: tedalanchong@163.com
}

Keywords: Experimental equipment, Electrostatics, Designing and building.

\begin{abstract}
High resistance flammable liquid electrostatics is the typical ignition source of industrial electrostatic fire explosion accidents, and thus understanding and revealing the electrostatic nature is very important for the electrostatic protection. On the basis of the fully research of liquid electrostatic discharge types and laws, the liquid electrostatic equipment is designed. This device can measure oil surface potential, and study the relationship between the oil velocity and the electrostatic charge, and investigate the relationship between oil surface potential and the discharge energy, and explore the radial distribution of oil surface potential.
\end{abstract}

\section{Introduction}

During the transmission and application of refined petroleum products, electrostatic accidents often happen. When speed of electrostatic accumulation is faster than speed of electrostatic dissipation, and this can cause electrostatic accumulation in petroleum [1,2]. When static electricity accumulates to a level, electrostatic discharge (ESD) happens under certain conditions, which can cause fires and explosions. Recently, with the increasing in the speed of oil injection, such causes electrostatic accumulation quickly and may increase the risk of ESD causing accidents [3,4]. It is necessary to research oil circulation system for static electric charges, surface potential of oil when ESD occurs and discharge energy. Therefore, a new experimental equipment for solving this problem was developed in the current work.

\section{Designing of Electrostatic Experimental Equipment}

On the basis of domestic and international research progress [5-8], experimental equipment of measuring liquid static charges is designed and developed. How to create static electric charges and store them is taken a full consideration. The equipment is made up of liquid circulation system and measuring support, which can measure the electrostatic potential of oil surface, investigate the relationship between flow rate and electrostatic accumulation, the relationship between filter material and electrostatic accumulation, the radial distribution of the electrostatic potential of oil surface and some other liquid static experiment.

Liquid Circulation System. The design diagram of the oil circulation system is shown in Fig. 1. The left side of Fig. 1 is oil drums, overflow tank and storage tank from top to bottom, respectively. And the right side of Fig. 1 from top to bottom is filter and oil pump, respectively. Two ball valves are installed between storage tank and pump (as shown in Fig. 2).

Cycle is as follows: at first, the storage tank is filled with kerosene, and then the oil pump is opened. Finally, kerosene is barreled into the test tank through the filter. Along with the rising of the oil level, the oil will overflow into the bottom of the overflow tank. A return pipe is designed in the bottom of the overflow groove, which can make the spilled oil to flow into the storage tank and then in a loop. A tee is installed between the pump and filter and the ball valve is installed between the tee and storage tank on the road. The above design is to control oil flow rate by controlling the open degree of the ball valve. This is called back design.

In order to have a good experimental effect, we should give full consideration to the charge generation and dissipation. In the circulatory system, the flow of oil will produce a static charge, but the electrostatic charge will be less in the current equipment because the volume of experimental 
equipment is relatively small. Therefore, we add the filter into the device in order to produce the static electricity as much as possible. In addition, we adopt insulation design methods to reduce the leakage of electrostatic charge, which make the static charge be preserved and measured. The insulation brace is designed at the bottom of the barrel and the overflow tank. And the rubber mat is used as insulating materials in the side of the overflow tank and pipe joint.

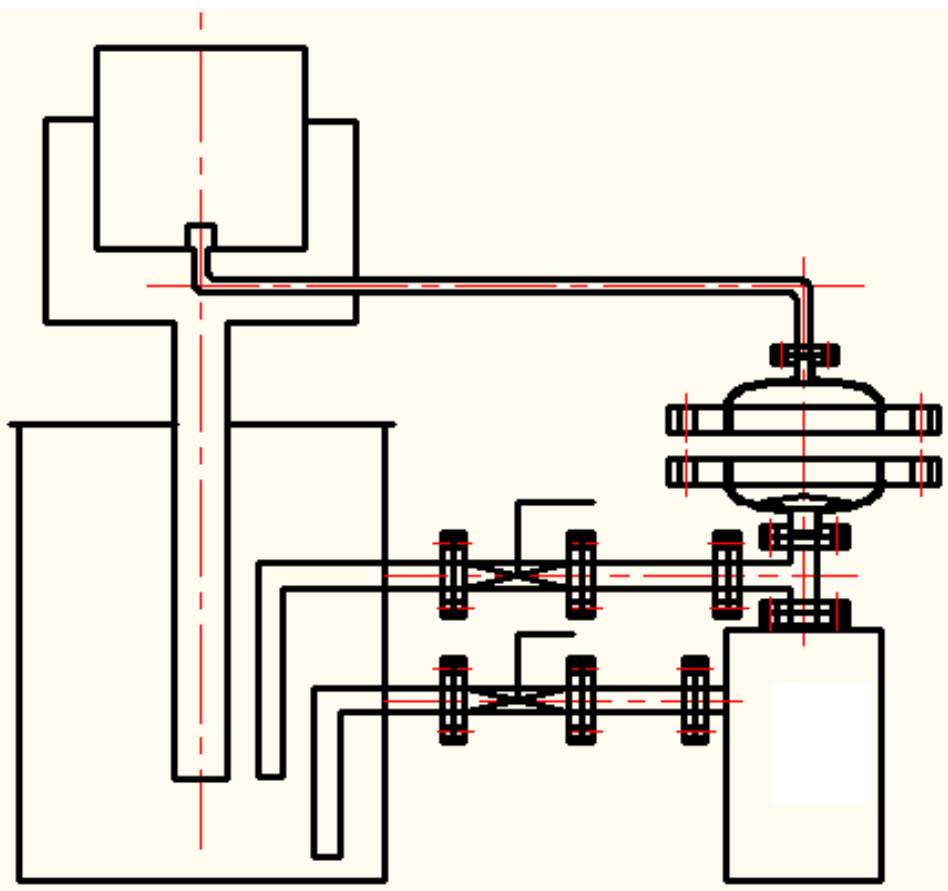

Fig. 1 Schematic diagram of oil circulation system

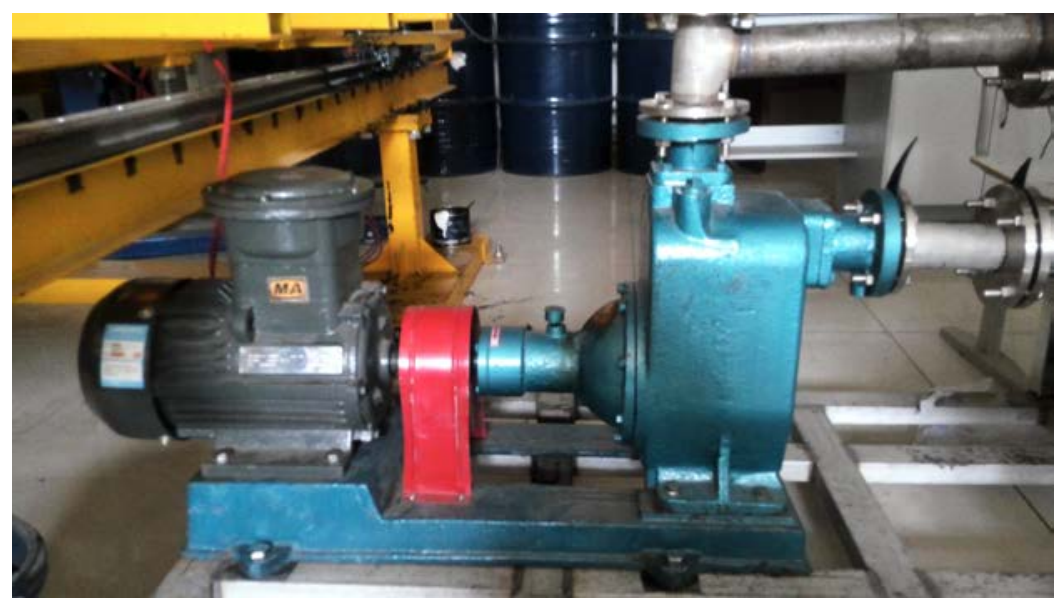

Fig. 2 50CYZ-50 oil pump

Measuring Support. Some liquid electrostatic related experiments can be carried out through the device, which mainly includes the measurement of oil surface potential and discharge energy. Therefore, two slider electrodes have been designed. The electrode is suspended under the slider, as shown in Fig. 3.

The upper part of Fig. 3 is the main view. And the under part of Fig. 3 is the top view, which is processed by a local section. The parts in the electrode frame design are numbered 1, 2, 3, 4 and 5, respectively. They are named as vertical plate, crossing slippery course, cylindrical rod, cylindrical rod handle and sliding block with electrodes.

The vertical plate is used as fixed supporting. And the cross slide rail is fixed on the vertical plate and used as the slide way of the electrode sliding block, which makes the electrode reciprocate on the 
cross road slippery course. Cylindrical rod connected with the electrode slider enables the slider reaches the measuring position.

Specific operation as follows: the vertical plate is fixed on the outside of the overflow tank. And then the cylinder is pulled to move the electrode to a measuring position. Finally, the electrostatic measurement is carried out.

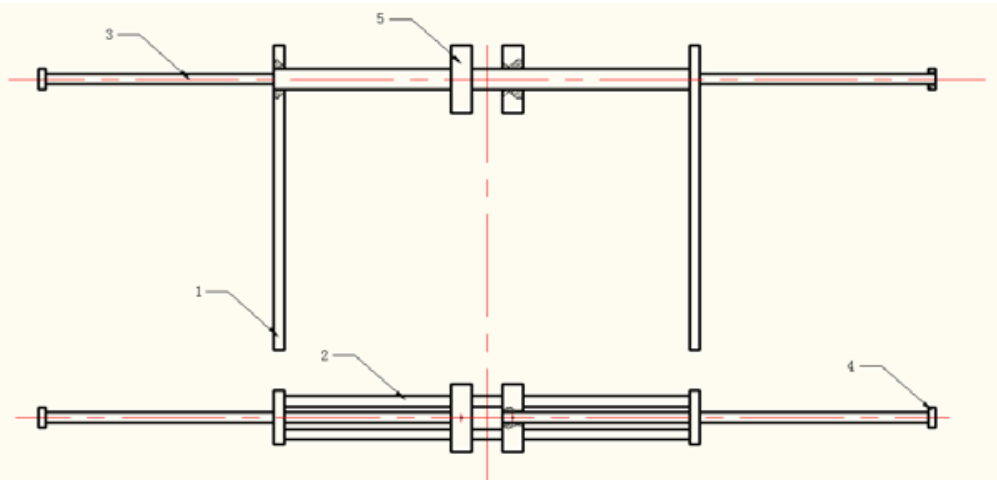

Fig. 3 Schematic diagram of measuring support

\section{Building of Electrostatic Experimental Equipment}

The experimental equipment designed is built to the bedstand, which can be applied to measure static charge. All joints of the equipment are sealed. The final test apparatus is shown in Fig. 4.

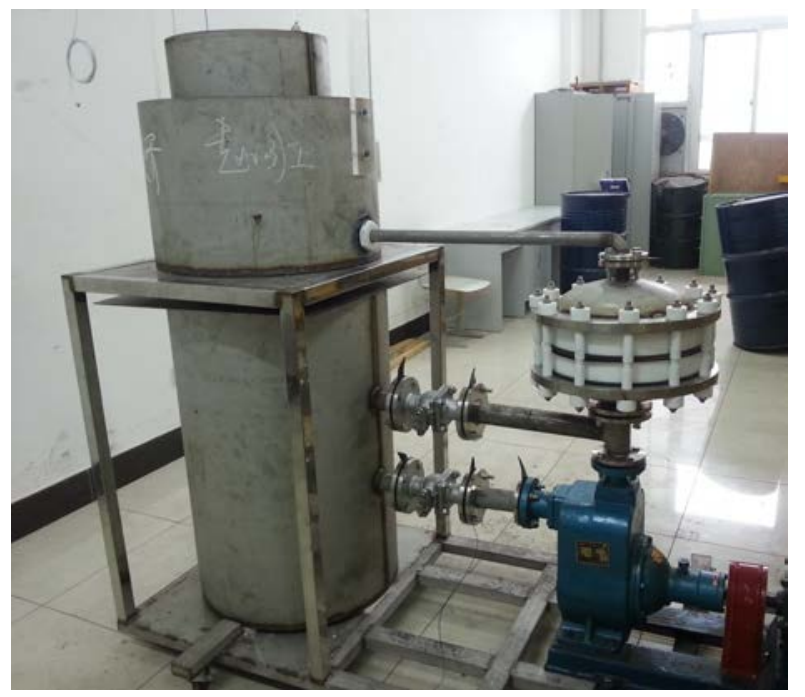

Fig. 4 Experimental equipment diagram

\section{Summary}

On the basis of the design, the liquid experimental equipment built can realize the circulation of liquid, and bracket can be used to the actual measurement of electrostatic charge that is generated through the filter. Both well-behaved insulation and sealing design are realized. The designed liquid electrostatic test apparatus can be applied for the measurement of electrostatic related experiments. Therefore, that is to say that the current experimental equipment is successfully designed and built.

\section{Acknowledgements}

This work was financially supported by the Undergraduate Innovation and Entrepreneurship Training Program of Liaoning Province (201310143026). 


\section{References}

[1] J. Gavis, I. Koszman. Development of charge in low conductivity liquids flowing past surfaces [J]. Journal of colloid science, 1961, 16: 375-391

[2] H. Walmsley. The calculation of the electrostatic potentials that occur when tanks are filled with charged liquids [J]. Journal of Electrostatics, 1991, 26(3): 201-226

[3] R.J. Pazada, T.B. Jones. Effect of surface conduction on charge relaxation in partially filled vessel [j]. Journal of Electrostatics, 1992, 28: 175-185

[4] J.X. Yang. Influence of injected charge on streaming electrification [J]. IEEE Trans. On Dielectrics Insulating, 1998, 5: 513-517

[5] G. Martin. Hazards and problems associated with liquids [J]. Journal of Electrostatics, 2001, 51-52: 359-365

[6] T.J. Harvey, R.J.K. Wood, G. Denuault, et al. Effect of oil quality on electrostatic charge generation and transport [J]. Journal of Electrostatics, 2002, 55(1): 1-23

[7] M. Giles. Electrostatic hazards in liquids and relevance to process chemistry [J]. Organic process research \& development, 2003, 7(6): 1048-1050

[8] M. Glor. Electrostatic ignition hazards in the process industry [J]. Journal of Electrostatics, 2005, 63(6-10): 447-453 